Rudyard Arteaga Solórzano; Freddy Mendoza Rivadeneira; Cecilia Párraga Alava; Plinio Vargas Zambrano

http://dx.doi.org/10.35381/r.k.v4i8.531

\title{
Elaboración, aplicación y evaluación de pesticidas orgánicos en jardín ornamental
}

\section{Development, application and evaluation of organic pesticides in ornamental garden}

Rudyard Arteaga Solórzano

r arteaga@utm.edu.ec

Universidad Técnica de Manabí, Portoviejo

Ecuador

https://orcid.org/0000-0001-5499-7334

Freddy Mendoza Rivadeneira

famendoza@utm.edu.ec

Universidad Técnica de Manabí, Portoviejo

Ecuador

https://orcid.org/0000-0003-1457-688X

Cecilia Párraga Alava

rparraga@utm.edu.ec

Universidad Técnica de Manabí, Portoviejo

Ecuador

https://orcid.org/0000-0003-1546-111X

Plinio Vargas Zambrano

pavargas@utm.edu.ec

Universidad Técnica de Manabí, Portoviejo

Ecuador

https://orcid.org/0000-0002-2152-7317

Recibido: 15 de mayo de 2019

Aprobado: 13 de junio de 2019

\section{RESUMEN}

El presente artículo tuvo como objetivo principal de elaborar, aplicar y evaluar pesticidas orgánicos en el jardín ornamental de la facultad de Ciencias Zootécnicas de la Universidad Técnica de Manabí. Para lo cual se tuvo como muestra poblacional ocho insectos que fueron observados en cuatro mediciones, así como la generación de 12 


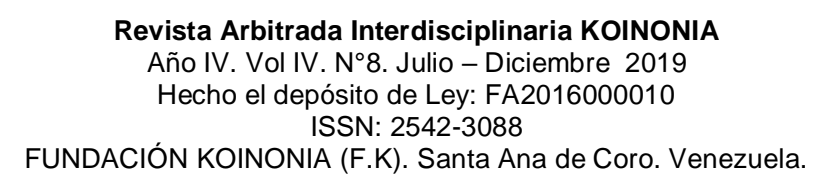

Rudyard Arteaga Solórzano; Freddy Mendoza Rivadeneira; Cecilia Párraga Alava; Plinio Vargas Zambrano

pesticidas orgánicos que fueron aplicados para el control de los insectos, así como la prevención implícita de plagas, hongos, que podrían estar presentes. En promedio de porcentaje se evidencia que los insecticidas que han presentados mejor evidencia de efectividad se encuentran: Ajo, Cebolla, Helecho, Neem, Tabaco. Lo cual conlleva a deducir sobre las propiedades que presentan en relación a la acción protectora en favorecer la erradicación de insectos en espacios de jardinería ornamental. La investigación permite corroborar que el uso de insecticidas biológicos, pueden ser concomitantes en la erradicación de plagas y de insectos.

Descriptores: Ecología; ecosistema; medio ambiente natural; efectos de las actividades humanas.

\section{ABSTRACT}

The purpose of this article was to elaborate, apply and evaluate organic pesticides in the ornamental garden of the Faculty of Zootechnical Sciences of the Technical University of Manabí. To this end, eight insects that were observed in four measurements were taken as a population sample, as well as the generation of 12 organic pesticides that were applied for the control of insects, as well as the implicit prevention of pests, fungi, which could be present. On average, it is evident that the insecticides that have presented the best evidence of effectiveness are: Garlic, Onion, Fern, Neem, Tobacco. This leads to deduce about the properties they present in relation to the protective action in favoring the eradication of insects in ornamental gardening spaces. The research allows us to confirm that the use of biological insecticides can be concomitant in the eradication of pests and insects.

Descriptors: Ecology; Ecosystems; Natural environment; Human activities effects.

\section{INTRODUCCIÓN}

Los pesticidas orgánicos son una opción para los jardineros que están preocupados por no dañar el ambiente y plantas de jardín con el uso de químicos sintéticos. Aunque están hechos con ingredientes que se presentan naturalmente, los pesticidas orgánicos pueden ser tóxicos, dañinos, para las mascotas y humanos. Esto es así porque muchos aún contienen químicos, a pesar de que deriven de fuentes naturales. Los pesticidas orgánicos no siempre son inocuos, pero cuando se eligen cuidadosamente, existen opciones mucho menos dañinas que sus equivalentes químicos.

A pesar de su base natural, los pesticidas orgánicos usualmente son tan efectivos como 


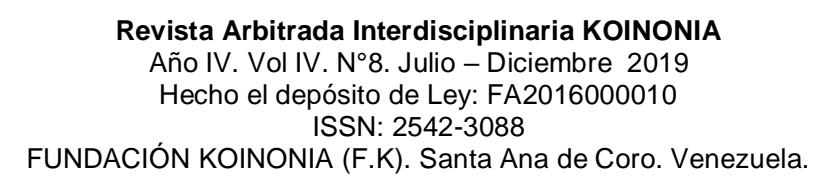

Rudyard Arteaga Solórzano; Freddy Mendoza Rivadeneira; Cecilia Párraga Alava; Plinio Vargas Zambrano

los químicos sintéticos para repeler plagas y proteger las plantas del jardín. Atacan con efectividad las plagas de insectos cuando se utilizan como se indica, ayudan a proteger el jardín de mayores daños. No obstante, en algunos casos, prevé aplicar más de una vez para controlar con efectividad los insectos. Los pesticidas orgánicos no tienen que ser comprados en una tienda de jardinería. En muchos casos, se puede contar en la alacena de la cocina los ingredientes vitales necesarios para preparar un líquido efectivo. Los pesticidas orgánicos también están disponibles con diferentes fórmulas, cada una de las cuales está diseñada para atacar un problema específico. Los productos químicos comerciales en raras ocasiones son específicos y suelen ser tóxicos para todos los insectos de jardín y vegetación. A diferencia de los productos químicos, existen pesticidas orgánicos que repelen a los insectos en vez de destruirlos.

Estos son particularmente atractivos para los jardineros que quieren repeler a los insectos en vez de destruirlos. Los productos con ingredientes como ajo u ortiga son repelentes de insectos efectivos para los jardines. La preparación se llevó a cabo en las áreas verdes de la Facultad de Ciencias Zootécnicas con estudiantes de la cátedra Biotecnología 3 y la efectividad fue puesta a prueba en plantas del jardín ornamental de la misma.

\section{MATERIALES Y MÉTODOS}

El proceso para obtener los principios activos depende del tipo de planta y de los componentes que se deseen extraer:

DECOCCIÓN: Hervir el agua con las plantas o partes desmenuzadas, durante 20 o 30 minutos. Se deja enfriar la decocción (tapada) y luego se filtra.

INFUSIÓN: Colocar agua hirviendo en un recipiente con las plantas o partes desmenuzadas. Tapar el recipiente y dejar en reposo 12 a 24 horas para luego filtrar el líquido.

ZUMO: Extraer el jugo de las plantas frescas, licuándolas, moliéndolas o machacándolas. Luego la papilla obtenida se coloca en una bolsa de tela para extraer el líquido a presión. MACERACIÓN: Las plantas desmenuzadas se colocan en un recipiente y se añade agua 


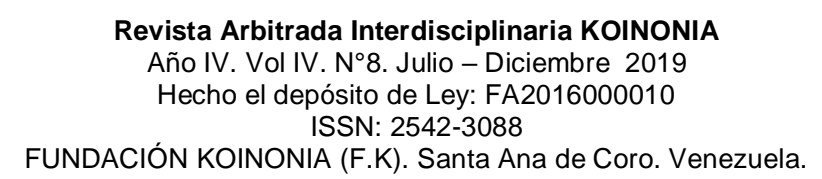

Rudyard Arteaga Solórzano; Freddy Mendoza Rivadeneira; Cecilia Párraga Alava; Plinio Vargas Zambrano

fría. La maceración dura de 1 a 2 días, transcurridos los cuales se filtra el líquido.

PURÍN FERMENTADO: Se colocan las plantas frescas en un recipiente de cerámica o madera; se añade agua y se tapa de tal manera que entre aire. Hay que remover diariamente la mezcla a fin de favorecer la fermentación. Cuando el líquido se ponga oscuro (aproximadamente dos semanas), sí ya no hace espuma al removerlo, se encuentra listo para filtrarlo y ser utilizado.

Para evaluar la efectividad se tomó en cuenta la observación de presencia de los insectos a controlar como un $100 \%$ antes de su aplicación y luego desde las respectivas aplicaciones cada dos días por cuatro ocasiones.

Es de destacar que se generó la investigación con el propósito de elaborar, aplicar y evaluar pesticidas orgánicos en el jardín ornamental de la facultad de Ciencias Zootécnicas de la Universidad Técnica de Manabí

\section{RESULTADOS}

\section{Cuadro 1}

Observación de presencia de insectos en jardín ornamental

\begin{tabular}{lrrrrr} 
Insectos & Día 1 & Día 4 & Día 7 & Día 10 & Total observados \\
\hline Hormigas & 5 & 2 & 2 & 4 & 13 \\
$\begin{array}{l}\text { Fumagina } \\
\text { Insectos minadores de }\end{array}$ & 4 & 3 & 3 & 5 & 15 \\
hojas & 5 & 4 & 4 & 3 & 16 \\
Ácaros & 3 & 5 & 5 & 5 & 18 \\
Áfidos & 4 & 3 & 5 & 4 & 16 \\
Chinches de ala de encaje & 3 & 5 & 4 & 5 & 17 \\
Arañas & 3 & 3 & 5 & 4 & 15 \\
\hline
\end{tabular}

Antes de la aplicación de los insecticidas orgánicos, se generó un proceso de observación con la intención de identificar los insectos que hacen presencia en el jardín ornamental de la facultad de Ciencias Zootécnicas de la Universidad Técnica de Manabí, destacándose que se generaron 4 observaciones, lo cual permitió medir la frecuencia de aparición y contar con una visión sobre el tratamiento a aplicar, para lo cual fue necesario 


\section{Revista Arbitrada Interdisciplinaria KOINONIA \\ Año IV. Vol IV. N8. Julio - Diciembre 2019 \\ Hecho el depósito de Ley: FA2016000010 \\ ISSN: 2542-3088 \\ FUNDACIÓN KOINONIA (F.K). Santa Ana de Coro. Venezuela.}

Rudyard Arteaga Solórzano; Freddy Mendoza Rivadeneira; Cecilia Párraga Alava; Plinio Vargas Zambrano

segmentar el jardín en 12 espacios, procediendo a la aplicación de los 12 insecticidas con la finalidad de observar la disminución o permanencia de los insectos.

\section{Cuadro 2}

Datos para los 12 pesticidas orgánicos

$\begin{array}{cccc}\text { Nombre común } & \text { Partes utilizadas } & \text { Principios activos } & \text { Plagas que } \\ \text { y científico } & \text { Extracción y } & \text { Que contiene } & \text { Controla } \\ & \text { fórmula } & & \end{array}$

\begin{tabular}{|c|c|c|c|}
\hline $\begin{array}{ll}\text { AJí- } & \text { Capsicum } \\
\text { annuum } & \end{array}$ & $\begin{array}{l}\text { Tallos tiernos y } \\
\text { follaje, } 100 \text { gr/litro, } \\
\text { mediante purín, } \\
\text { decocción } \\
\text { infusión }\end{array}$ & $\begin{array}{l}\text { Capsaicina, } \\
\text { carotenoides, } \\
\text { flavonoides, vitamina } \\
\text { C }\end{array}$ & $\begin{array}{l}\text { Pulgones, orugas, } \\
\text { hormigas }\end{array}$ \\
\hline AJO- Allium sativum & $\begin{array}{l}\text { Bulbo, } 1 \mathrm{~kg} \text { en } 100 \\
\mathrm{ml} \text { aceite de cocina } \\
\text { y } 1 / 2 \text { jabón para } \\
\text { maceración }\end{array}$ & $\begin{array}{l}\text { Aceite esencial } \\
\text { disulfuro de alilo, } \\
\text { alina, alisina, } \\
\text { vitaminas A y C, } \\
\text { nicotilamina, yodo }\end{array}$ & $\begin{array}{l}\text { Pulgones, orugas, } \\
\text { ácaros, gorgojos, } \\
\text { chinches, bacterias } \\
\text { y hongos }\end{array}$ \\
\hline $\begin{array}{l}\text { CEBOLLA- Allium } \\
\text { сера }\end{array}$ & $\begin{array}{l}\text { Bulbos, } 100 \text { gr/litro } \\
\text { para purín }\end{array}$ & $\begin{array}{l}\text { Aceites esenciales, } \\
\text { azúcares vitaminas, } \\
\text { compuestos } \\
\text { orgánicos sulfurados } \\
\text { (antisépticos) de } \\
\text { fuerte efecto } \\
\text { antibiótico }\end{array}$ & $\begin{array}{l}\text { Pulgones, ch } \\
\text { orugas }\end{array}$ \\
\hline $\begin{array}{l}\text { COLA } \\
\text { CABALLO- } \\
\text { Equisetum arvense }\end{array}$ & $\begin{array}{l}\text { allos y hojas, } 150 \\
\text { r/litro en } \\
\text { lecocción y purín }\end{array}$ & $\begin{array}{l}\text { Ácido cilísico, } \\
\text { saponina, glucósido } \\
\text { flavonólico, ácidos }\end{array}$ & $\begin{array}{l}\text { Pulgones, polillas, } \\
\text { ácaros, araña roja y }\end{array}$ \\
\hline
\end{tabular}




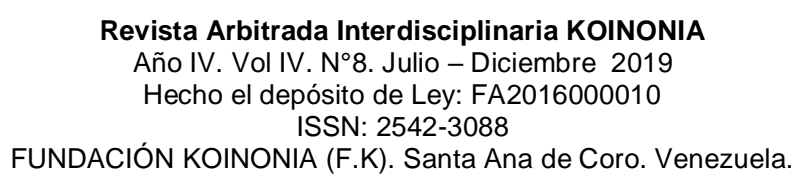

Rudyard Arteaga Solórzano; Freddy Mendoza Rivadeneira; Cecilia Párraga Alava; Plinio Vargas Zambrano

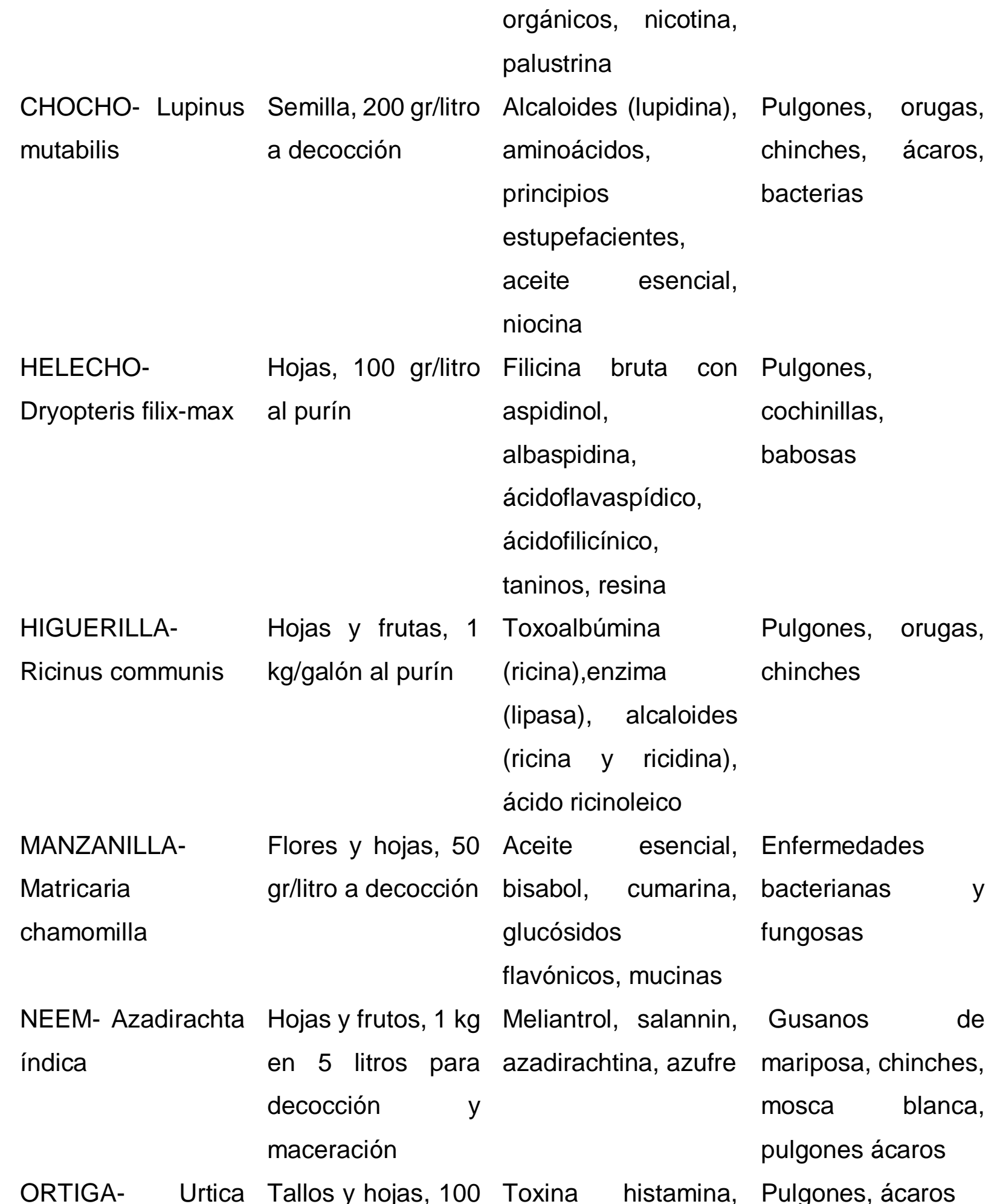




\section{Revista Arbitrada Interdisciplinaria KOINONIA \\ Año IV. Vol IV. №8. Julio - Diciembre 2019 \\ Hecho el depósito de Ley: FA2016000010 \\ ISSN: 2542-3088 \\ FUNDACIÓN KOINONIA (F.K). Santa Ana de Coro. Venezuela}

Rudyard Arteaga Solórzano; Freddy Mendoza Rivadeneira; Cecilia Párraga Alava; Plinio Vargas Zambrano

dioica

gr/litro

para ácido

fórmico,

maceración y purín glucoquinina

RÁBANO- Hojas y raíces, 30 Aceite esencial Monilia y bacterias

Armoracia rusticana gr/litro para la (senevol) sustancias

decocción bacteriostáticas y

antisépticas

TABACO- Nicotiana Hojas, 50 gr/litro Nicotina

Pulgones, moscas, tabacum

para infusión chinches, orugas, ácaros

Se trabajó con los doce pesticidas orgánicos elaborados por los investigadores y descritos en el cuadro 1, con la finalidad de generar un proceso no solo de elaboración, sino de aplicación en un lapso de 10 días con la finalidad de observar y evaluar los avances generados mediante la aplicación de los diversos insecticidas.

\section{Cuadro 3}

Porcentaje de efectividad por insecticida orgánico

\begin{tabular}{|c|c|c|c|c|c|c|c|c|}
\hline Insectos & Día 1 & $\%$ & Día 4 & $\%$ & Día 7 & $\%$ & Día 10 & $\%$ \\
\hline Ají & 2 & 8 & 1 & 4 & 3 & 8 & 2 & 4 \\
\hline Ajo & 3 & 12 & 2 & 8 & 4 & 10 & 3 & 6 \\
\hline Cebolla & 2 & 8 & 3 & 11 & 5 & 13 & 4 & 9 \\
\hline Cola de caballo & 1 & 4 & 4 & 15 & 2 & 5 & 3 & 6 \\
\hline Chocho & 2 & 8 & 2 & 7 & 3 & 8 & 5 & 11 \\
\hline Helecho & 3 & 12 & 3 & 11 & 4 & 11 & 4 & 9 \\
\hline Higuerilla & 2 & 8 & 1 & 4 & 2 & 5 & 5 & 11 \\
\hline Manzanilla & 1 & 4 & 2 & 7 & 3 & 8 & 4 & 9 \\
\hline Neem & 2 & 8 & 3 & 11 & 4 & 11 & 5 & 11 \\
\hline Ortiga & 1 & 4 & 1 & 4 & 2 & 5 & 3 & 6 \\
\hline Rábano & 2 & 8 & 2 & 7 & 1 & 3 & 5 & 11 \\
\hline Tabaco & 4 & 16 & 3 & 11 & 5 & 13 & 3 & 7 \\
\hline
\end{tabular}




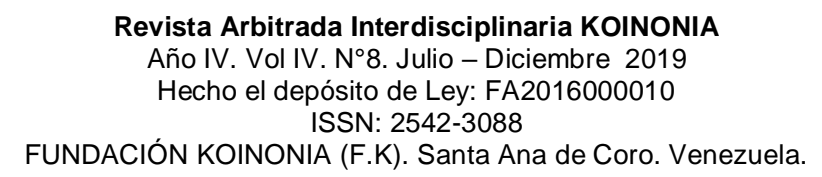

Rudyard Arteaga Solórzano; Freddy Mendoza Rivadeneira; Cecilia Párraga Alava; Plinio Vargas Zambrano

Se presentan los 12 insecticidas por día de tratamiento, teniéndose que el ají para el día 1, presentó una efectividad de $8 \%$, día 2 un $4 \%$ de efectividad, día 7 una efectividad de $8 \%$ y para día 10 un $4 \%$

El Ajo para el día 1, presentó una efectividad de 12\%, día 2 un $8 \%$ de efectividad, día 7 una efectividad de $10 \%$ y para día 10 un $6 \%$

La cebolla para el día 1, presentó una efectividad de $8 \%$, día 2 un $11 \%$ de efectividad, día 7 una efectividad de 13\% y para día 10 un $9 \%$

La cola de caballo para el día 1, presentó una efectividad de 4\%, día 2 un 15\% de efectividad, día 7 una efectividad de $5 \%$ y para día 10 un 6\%

El Chocho para el día 1, presentó una efectividad de 8\%, día 2 un 7\% de efectividad, día 7 una efectividad de $8 \%$ y para día 10 un $11 \%$

El Helecho para el día 1, presentó una efectividad de 12\%, día 2 un $11 \%$ de efectividad, día 7 una efectividad de $11 \%$ y para día 10 un 9\%

La Higuerilla para el día 1, presentó una efectividad de $8 \%$, día 2 un 4\% de efectividad, día 7 una efectividad de 5\% y para día 10 un $11 \%$

La manzanilla para el día 1, presentó una efectividad de 4\%, día 2 un 7\% de efectividad, día 7 una efectividad de $8 \%$ y para día 10 un 9\%

El Neem para el día 1, presentó una efectividad de 8\%, día 2 un $11 \%$ de efectividad, día 7 una efectividad de $11 \%$ y para día 10 un $11 \%$

La Ortiga para el día 1, presentó una efectividad de 4\%, día 2 un 4\% de efectividad, día 7 una efectividad de $5 \%$ y para día 10 un $6 \%$

El Rábano para el día 1, presentó una efectividad de 8\%, día 2 un 7\% de efectividad, día 7 una efectividad de $3 \%$ y para día 10 un $11 \%$

El Tabaco para el día 1, presentó una efectividad de 16\%, día 2 un 11\% de efectividad, día 7 una efectividad de 13\% y para día 10 un 7\% 


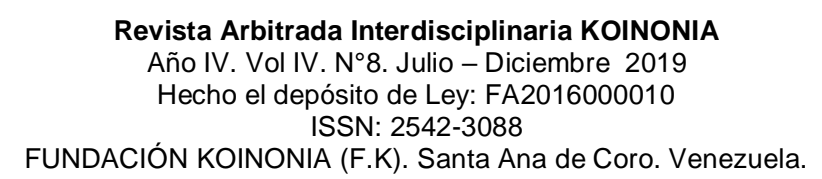

Rudyard Arteaga Solórzano; Freddy Mendoza Rivadeneira; Cecilia Párraga Alava; Plinio Vargas Zambrano

En promedio de porcentaje se evidencia que los insecticidas que han presentados mejor evidencia de efectividad se encuentran: Ajo, Cebolla, Helecho, Neem, Tabaco. Lo cual conlleva a deducir sobre las propiedades que presentan en relación a la acción protectora en favorecer la erradicación de insectos en espacios de jardinería ornamental.

\section{CONCLUSIONES}

Los insecticidas naturales también representan riesgos y beneficios, los cuales es necesario considerar, así como sus formas de uso.

Numerosos químicos se producen naturalmente y funcionan en algún grado como insecticidas. Están presentes en la mayoría de los organismos vivos, desde las algas azul-verdes, hongos y las angiospermas.

Los compuestos son tan variados como las plantas de las cuales han sido aislados y el rango de su efecto protector va desde repelencia, disuasión de la alimentación y oviposición hasta toxicidad aguda e interferencia con el crecimiento y el desarrollo de los insectos.

Los insecticidas vegetales presentan la gran ventaja de ser compatibles con otras opciones de bajo riesgo aceptables en el control de insectos, tales como feromonas, aceites, jabones, hongos entomopatógenos, depredadores y parasitoides, entre otros, lo que aumenta enormemente sus posibilidades de integración a un programas de Manejo Integrado de Plagas.

La actividad biológica de un compuesto natural está en función de su estructura y en la dosis usada para tales fines.

La investigación permite corroborar que el uso de insecticidas biológicos, pueden ser concomitantes en la erradicación de plagas y de insectos, cuando se genera un adecuado procesamiento y aplicación de los mismos, lo cual el procedimiento generado podría ser empleado por investigadores en diversas áreas investigativas con la finalidad de conocer el comportamiento de los insecticidas en otros entornos ambientales, siendo necesaria además la realización de nuevas experimentaciones con la finalidad de observar y cotejar 


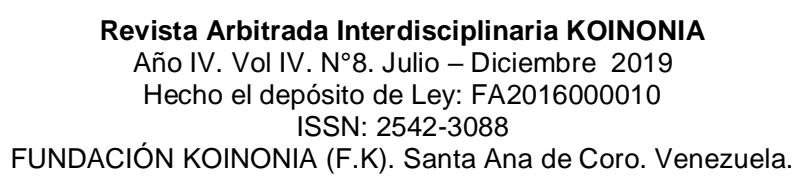

Rudyard Arteaga Solórzano; Freddy Mendoza Rivadeneira; Cecilia Párraga Alava; Plinio Vargas Zambrano

si existió erradicación total o temporal de los insectos abordados en el actual papel de trabajo.

\section{REFERENCIAS CONSULTADAS}

1. Carpinella, M. Defago, M. T. Valladares, G. And Palacios S. M. (2003). Antifeedant and insecticide properties of a Limonoid from Melia azedarach (Meliaceae) with potential use for pest management. J. Agric. Food Chem. 51, 369-374

2. Céspedes, C. L., Calderón, J. S., Lina, L. and Aranda, E. (2000). Growth effects on fall armyworm Spodoptera frugiperda of some limonoids isolated from Cedrela spp. (Meliaceae). J. Agric. Food Chem. 48, 1903-1908.

3. Silva, G., Lagunes, A., Rodríguez, J. C., y Rodríguez, D. (2002). Insecticidas vegetales; Una vieja-nueva alternativa en el control de plagas. Revista Manejo Integrado de Plagas (CATIE).

4. Suresh, G., Geetha Gopalakrishman; Daniel Wesley, S.; Pradeep N. D.; Malathi, R. and Rajan, S. S. (2002). Insect antifeedant activity of tetranortriterpenoids from the rutales. A perusal of structural relations. J. Agric. Food Chem., 50, 4484-4490.

\section{REFERENCES CONSULTED}

1. Carpinella, M. Defago, M. T. Valladares, G. And Palacios S. M. (2003). Antifeedant and insecticide properties of a Limonoid from Melia azedarach (Meliaceae) with potential use for pest management. J. Agric. Food Chem. 51, 369-374

2. Céspedes, C. L., Calderón, J. S., Lina, L. and Aranda, E. (2000). Growth effects on fall armyworm Spodoptera frugiperda of some limonoids isolated from Cedrela spp. (Meliaceae). J. Agric. Food Chem. 48, 1903-1908.

3. Silva, G., Lagunes, A., Rodríguez, J. C., and Rodríguez, D. (2002). Plant insecticides; An old-new alternative in pest control. Integrated Pest Management Magazine (CATIE).

4. Suresh, G., Geetha Gopalakrishman; Daniel Wesley, S .; Pradeep N. D .; Malathi, R. and Rajan, S. S. (2002). Insect antifeedant activity of tetranortriterpenoids from the rutales. A perusal of structural relations. J. Agric. Food Chem., 50, 4484-4490. 
Revista Arbitrada Interdisciplinaria KOINONIA

Año IV. Vol IV. №8. Julio - Diciembre 2019

Hecho el depósito de Ley: FA2016000010

ISSN: 2542-3088

FUNDACIÓN KOINONIA (F.K). Santa Ana de Coro. Venezuela.

Rudyard Arteaga Solórzano; Freddy Mendoza Rivadeneira; Cecilia Párraga Alava; Plinio Vargas

Zambrano

(C2019 por lo autores. Este artículo es de acceso abierto y distribuido según los términos y condiciones de la licencia Creative Commons Atribución-NoComercial-Compartirlgual 4.0 Internacional (CC BY-NC-SA 4.0) (https://creativecommons.org/licenses/by-nc-sa/4.0/). 